Liver, Pancreas and Biliary Tract

\title{
Epidemiological and clinical scenario of chronic liver diseases in Italy: Data from a multicenter nationwide survey
}

\author{
Evangelista Sagnelli ${ }^{a, *}$, Tommaso Stroffolini $^{b}$, Caterina Sagnelli $^{c}$, Antonina Smedile $^{\mathrm{d}}$, \\ Filomena Morisco $^{\mathrm{e}}$, Caterina Furlan ${ }^{\mathrm{b}}$, Sergio Babudieri ${ }^{\mathrm{f}}$, Giuseppina Brancacciog ${ }^{\mathrm{g}}$, \\ Nicola Coppola ${ }^{a}$, Giovanni Battista Gaetag, \\ Piero Luigi Almasio $^{\mathrm{h}}$, on behalf of the EPACRON study Group ${ }^{1}$
}

a Department of Mental Health and Public Medicine, Second University of Naples, Italy

${ }^{\mathrm{b}}$ Department of Tropical and Infectious Diseases, Policlinico Umberto Primo, Rome, Italy

${ }^{\mathrm{c}}$ Department of Clinical and Experimental Medicine and Surgery, Second University of Naples, Napoli, Italy

d Department of Medical Sciences, University of Turin, Turin, Italy

e Department of Clinical Medicine and Surgery, Gastroenterology Unit, University of Naples "Federico II", Napoli, Italy

${ }^{\mathrm{f}}$ Clinic of Infectious Diseases, University of Sassari, Sassari, Italy

${ }^{g}$ Department of Clinical and Experimental Medicine and Surgery, Viral Hepatitis Unit, University of Naples, Napoli, Italy

${ }^{\mathrm{h}}$ Gastroenterology \& Hepatology Unit, Di.Bi.MI.S. University of Palermo, Palermo, Italy

\section{A R T I C L E I N F O}

\section{Article history:}

Received 13 February 2016

Accepted 16 May 2016

Available online 26 May 2016

\section{Keywords:}

Chronic hepatitis

Chronic liver diseases

HCC

Liver cirrhosis

\begin{abstract}
A B S T R A C T
Background: The last Italian prevalence survey on chronic liver diseases (CLD) was performed in 2001. The present study evaluated the changes occurring over thirteen years.

Methods: We enrolled 2,557 CLD consecutive patients in 16 Italian liver units in 2014.

Results: HBV etiology accounted for 513 (20.2\%) cases, alone in 439 and associated with HCV and/or alcohol abuse in 74 . Of these 513, 11.9\% were anti-HDV-positive and 7.2\% HBeAg-positive. HCV alone was responsible for $50.3 \%$ of CLD and with alcohol abuse for $5.9 \%$. HCV RNA was detected in $64.0 \%$ of the anti-HCV-positive patients tested. HCV genotyping, performed for 899 patients, showed genotype$1 \mathrm{a}, 1 \mathrm{~b}, 2,3,4$ and 5 respectively in $16.5 \%, 45.5 \%, 15.4 \%, 8.2 \%, 15.1 \%$ and $0.2 \%$. Alcohol abuse alone was responsible for $6.4 \%$ of cases and NAFLD/NASH for $6.3 \%$. Liver cirrhosis $(p<0.001)$ and HCC $(p<0.001)$ were more frequent in alcoholic than viral etiologies. HCV and alcohol etiologies were more frequent in 2001 than 2014 (from $69.9 \%$ to $59.9 \%$ and from $23.0 \%$ to $12.3 \%$, respectively). HBV showed a similar impact. In all etiologies, the 2001 CLD cases were 10 years younger and with a significantly lower rate of cirrhosis than the 2014 cases.

Conclusion: The changes in HCV, HBV and alcohol etiologies may help apply more appropriate healthcare strategies.
\end{abstract}

(C) 2016 Editrice Gastroenterologica Italiana S.r.l. Published by Elsevier Ltd. All rights reserved.

\section{Introduction}

An Italian multicenter retrospective study performed on 1,154 patients with chronic liver diseases (CLD) enrolled from 1976 to 1981 showed that $60 \%$ of the cases were HBsAg-positive and the

\footnotetext{
* Corresponding author at: Department of Mental and Public Health, Section of Infectious Diseases, Second University of Naples, Largo Madonna delle Grazie n. 1, Naples, Italy. Tel.: +390815666503/0815666508.

E-mail addresses: evangelista.sagnelli@unina2.it, evangelistasagnelli@libero.it (E. Sagnelli)

1 Other authors from the operative units: Angelo Andriulli, Bruno Cacopardo, Guido Colloredo, Massimo De Luca, Mario Pirise, Mariantonietta Pisaturo, Maurizio Russello, Floriano Rosina, Teresa Santantonio.
}

remaining $40 \% \mathrm{HBsAg}$-negative. In the absence of a specific test for $\mathrm{HCV}$ etiology, the HBsAg-negative cases were attributed to a NonA Non-B agent [1] and other etiologies remained unexplored. HBV etiology was observed in $31.3 \%$ of 5,461 cases with CLD studied from 1980 to 1989 [2] in another Italian multicenter prevalence survey in which $46.4 \%$ of the cases were attributed to a Non-A Non-B agent, $3 \%$ to an autoimmune etiology, $10.8 \%$ to alcohol abuse and $2.9 \%$ to other less frequent etiologies. An HBV/HDV multiple infection was diagnosed in $5.5 \%$ of the HBsAg-positive cases.

A further impressive decline in HBV etiology was observed in a prospective multicenter investigation on 9,997 Italian patients with chronic liver disease studied in 79 liver units in 2001 [3]. In this study, $10 \%(n=1,000)$ of the cases were due to HBV infection alone, $1.4 \%(n=140)$ were HBV/alcohol-related, $1.4 \%(n=141)$ 
HBV/HCV-related and 0.6\% ( $n=63) \mathrm{HBV} / \mathrm{HCV} /$ alcohol-related. In addition, $56.3 \%(n=5,632)$ of the cases were due to HCV infection alone, $11.6 \%(n=1,163)$ were $\mathrm{HCV} /$ alcohol-related, 9.4\% $(n=935)$ alcohol-related, $0.8 \%(n=76)$ autoimmune, $0.5 \%(n=25)$ PBC, $4.4 \%$ $(n=444)$ NALFD and the remaining $3.6 \%$ were attributed to other infrequent etiologies. An HBV/HDV multiple infection was detected in $9.7 \%$ of the $\mathrm{HBsAg}$-positive cases. These prevalences remained substantially unchanged when only the 6,210 patients with chronic hepatitis were evaluated [4]. These data indicate a decrease in the rate of HBV-related cases from 1976 to 2001 and a corresponding increase in the rate of the HCV-related cases.

The lack of information on these trends after 2001 induced us to conduct a new prospective multicenter prevalence survey on the etiology of CLD in Italy.

\section{Materials and methods}

\subsection{Study population and methods}

The present study, named the EPACRON study, recruited 2,557 patients with CLD from January to December 2014. The enrolling criteria were age over 18 years and patients admitted for either altered hepatic biochemistry or presence of etiologic markers of liver diseases or referring symptoms consistent with CLD. Both in- and outpatients were consecutively admitted to one of 16 cooperating Liver Units located in different Italian regions. Of the 16 participating Liver Units, 7 were clinical centers of Departments of Infectious Diseases, 5 of Departments of Gastroenterology and 4 of Departments of Internal Medicine. Nearly two-thirds of patients were recruited in Liver Units of Departments of Gastroenterology or Internal Medicine and nearly one third in Liver Units of Departments of Infectious Diseases. Several of these centers have cooperated for a decade in numerous clinical investigations applying the same clinical approach and similar analytical methods [5-7].

The collection of personal data was made in full compliance with the Italian law on personal data protection, and each patient gave his/her informed consent to participate. All procedures applied in the study were in accordance with the international guidelines, with the standards of human experimentation of the local Ethics Committees and with the Helsinki Declaration of 1975, revised in 1983. At the time of the first observation, each patient signed their informed consent for the collection of personal data, as designated by the Ethics Committee of the coordinating center. Patients who agreed to undergo liver biopsy signed an appropriate informed consent before biopsy was performed. All patients were included only once, even if seen several times during the observation period. For each patient a pre-coded questionnaire containing demographic, epidemiological and clinical data was filled out. No patient refused to participate in the study.

The presence of serum HBsAg identified an HBV etiology, and the detection of anti-HCV an HCV etiology. Autoimmune chronic hepatitis and primary biliary cholangitis were diagnosed according to standardized international criteria [8-10]. The diagnosis of hereditary hemochromatosis was made on the basis of abnormal ferritin serum values and transferrin saturation serum values, genetic markers, or liver histology [8,11]. Wilson's disease was a rare diagnosis, made on the basis of accepted criteria $[8,12]$. The presence of a metabolic syndrome was established based on accepted criteria [8].

Abnormal serum alanine aminotransferase (ALT) values and a histological and/or ultrasound pattern of hepatic steatosis, in the absence of other known causes of chronic liver disease, were considered related to non-alcoholic fatty liver disease [13]. An alcohol intake $\geq 40 \mathrm{~g} /$ day for males and $\geq 30 \mathrm{~g} /$ day for females for at least 5 years was considered an etiologic factor of liver disease. A cryptogenic chronic liver disease was diagnosed in the absence of any viral, autoimmune or metabolic etiology. Chronic hepatitis was diagnosed on the basis of liver histology, when available, or on the persistence ( $>6$ months) of abnormal ALT in the absence of clinical, biochemical, and ultrasound markers of liver cirrhosis [8,14]. Liver cirrhosis was diagnosed by liver biopsy (LB) or on the presence of characteristic clinical, biochemical, and ultrasound signs [8,14]. The diagnosis of hepatocellular carcinoma (HCC) was based on histological and/or imaging findings and alfa-1-fetoprotein serum levels, according to accepted criteria $[8,15]$. Percutaneous LB was performed, if requested by the physician in care for diagnostic purposes, under US guidance using a disposable modified Menghini needle. In each liver unit a skilled pathologist unaware of the clinical and laboratory data evaluated liver histology. In particular, liver necroinflammation and fibrosis were assessed by the Ishak [16] or Metavir scoring system [17], and standardized criteria were used to convert the Ishak score to a Metavir score [18]. Transient elastometry was performed by Fibroscan [19,20]. Serum HBsAg and antibody to HCV, HDV and HIV were sought using commercial immunoenzymatic assays. Plasma HBV DNA was determined by real-time polymerase chain reaction (PCR) [21]; by this method, the detection limit in plasma samples is estimated at around $40 \mathrm{IU} / \mathrm{mL}$. HCV RNA was detected and quantified by a real-time PCR in a Light cycler 1.5 ; by this method, the detection limit in plasma samples is estimated at around $40 \mathrm{IU} / \mathrm{mL}$. HCV genotyping was performed using a commercial Line-Probe assay.

Routine tests were applied to seek the etiologic markers of autoimmune hepatitis, PBC, iron and copper overload and liver functions.

\subsection{Statistical analysis}

The data were collected in a pre-established electronic CRF database (web-based data collection, e-CRF provided by Air-Tel ${ }^{\circledR}$, Airon Telematica, Milan, Italy). Differences in the distribution of the characteristics of the subjects in the different groups were evaluated applying the analysis of variance and the Chi-square analysis for continuous and categorical variables, respectively. A $p$ value less than 0.05 was considered statistically significant. All $p$ values were two-tailed.

\section{Results}

At the end of the recruitment period, 2,557 patients with chronic liver diseases had been consecutively enrolled. Males predominated ( $n=1,512 / 59.1 \%$ ) and the majority of patients were of Italian/Caucasian origin $(n=2,420 / 94.7 \%)$. Less than one-third of the cases had a high school diploma or degree. Incidental finding on screening accounted for $80.7 \%(n=2,064)$ of the patients enrolled and $86.0 \%$ ( $n=2,200$ ) were observed as outpatients (Table 1$)$. Current alcohol abuse was stated by $12.0 \%(n=307)$ of the patients enrolled in the present study, past alcohol abuse by $18 \%(n=460)$, whereas $54 \%(n=1,381)$ were abstainers or "social drinkers"; no or doubtful information was given by the remaining $16 \%(n=409)$ (Table 1). Type- 1 diabetes was present in $4.5 \%(n=116)$ of patients and type-2 diabetes in $9.6 \%(n=245)$. Compared with the 2,200 outpatients, the 357 inpatients were older $(62.4 \pm 12.9$ vs. $58.3 \pm 14.0$, $p \leq 0.001)$, prevalently males $(n=228 / 63.9 \%$ vs. $n=1,284 / 58.4 \%$, $p=0.05)$ and more frequently showed decompensated cirrhosis or HCC ( $n=225 / 45.2 \%$ vs. $n=609 / 10.7 \%, p \leq 0.001$ ) (Table 2 ). Compared with inpatients, outpatients more frequently showed HBV $(n=396 / 18 \%$ vs. $n=43 / 12 \%)$ and HCV etiology $(n=1,119 / 50.9 \%$ vs. $n=167 / 46.8 \%$ ) and less frequently alcoholic ( $n=130 / 5.9 \%$ vs. $n=33 / 9.2 \%$ ) etiology and virus \pm alcohol (6.6\% of 146 vs. $13.4 \%$ of 
Table 1

General demographic features of the entire cohort.

\begin{tabular}{|c|c|}
\hline No of cases & 2,557 \\
\hline Mean age (range), years & $58.9 \pm 13.9(18-90)$ \\
\hline \multicolumn{2}{|l|}{ Gender, $N(\%)$} \\
\hline Male & $1,512(59.1)$ \\
\hline Female & $1,045(40.9)$ \\
\hline \multicolumn{2}{|l|}{ Country of origin, $N(\%)$} \\
\hline Italy & $2,420(94.7)$ \\
\hline Eastern Europe & $77(3.0)$ \\
\hline Rest of the world & $60(2.3)$ \\
\hline \multicolumn{2}{|l|}{ Education, $N(\%)$} \\
\hline Primary school & $658(25.7)$ \\
\hline Secondary school & $859(33.6)$ \\
\hline High school/University & $561(22.0)$ \\
\hline Not reported & $479(18.7)$ \\
\hline \multicolumn{2}{|l|}{ Mode of hospital admission, $N(\%)$} \\
\hline Outpatients & $2,200(86.0)$ \\
\hline Inpatients & $357(14.0)$ \\
\hline \multicolumn{2}{|l|}{ Referral pattern, $N(\%)$} \\
\hline Advice of general practitioner & $927(36.3)$ \\
\hline Self-referral & $780(30.5)$ \\
\hline Other & $558(21.7)$ \\
\hline Unknown & $292(11.5)$ \\
\hline \multicolumn{2}{|l|}{ Mode of detection, $N(\%)$} \\
\hline Blood donation & $63(2.5)$ \\
\hline Incidental on screening & $2,064(80.7)$ \\
\hline Liver disease in the family & $116(4.5)$ \\
\hline Unknown & $314(12.3)$ \\
\hline \multicolumn{2}{|l|}{ Alcohol intake, $N(\%)$} \\
\hline Abstainer & $1,381(54.0)$ \\
\hline Current & $307(12.0)$ \\
\hline Past & $460(18.0)$ \\
\hline Not reported & $409(16.0)$ \\
\hline \multicolumn{2}{|l|}{ With diabetes, $N(\%)$} \\
\hline Type 1 & $116(4.5)$ \\
\hline Type 2 & $245(9.6)$ \\
\hline
\end{tabular}

48) double etiology. The percentages of patients with NAFLD/NASH were $n=138 / 6.3 \%$ in outpatients and $n=24 / 6.7 \%$ in inpatients.

Table 3 shows the etiologies of chronic liver diseases registered in the present study (year 2014) and, for comparison, those found in the previous one performed in 2001. In the present study

Table 2

Comparison of 2,557 patients according to mode of hospital admission.

\begin{tabular}{|c|c|c|c|}
\hline Characteristics & $\begin{array}{l}\text { Inpatients } \\
(n=357)\end{array}$ & $\begin{array}{l}\text { Outpatients } \\
(n=2,200)\end{array}$ & $p$-value \\
\hline Mean age (range), years & $62.4 \pm 12.9$ & $58.3 \pm 14.0$ & $<0.001$ \\
\hline \multicolumn{4}{|l|}{ Gender, $N(\%)$} \\
\hline Male & $228(63.9)$ & $1,284(58.4)$ & \multirow[t]{2}{*}{0.05} \\
\hline Female & $129(36.1)$ & $916(41.6)$ & \\
\hline \multicolumn{4}{|l|}{ Country of origin, $N(\%)$} \\
\hline Italy & $347(97.2)$ & $2,073(94.2)$ & \multirow{3}{*}{0.03} \\
\hline Eastern Europe & $8(2.2)$ & $69(3.1)$ & \\
\hline Rest of the world & $2(0.6)$ & $58(2.6)$ & \\
\hline \multicolumn{4}{|l|}{ Diagnosis, ${ }^{\mathrm{a}} N(\%)$} \\
\hline Absent/mild liver injury & $1(0.3)$ & $10(0.6)$ & \multirow{5}{*}{$<0.001$} \\
\hline Chronic hepatitis & $95(29.6)$ & $1,129(64.6)$ & \\
\hline Compensated cirrhosis & $80(24.9)$ & $421(24.1)$ & \\
\hline Decompensated cirrhosis & $84(26.2)$ & $100(5.7)$ & \\
\hline $\mathrm{HCC}$ & $61(19.0)$ & $88(5.0)$ & \\
\hline \multicolumn{4}{|l|}{ With diabetes, $N(\%)$} \\
\hline Type 1 & $51(14.3)$ & $65(3.0)$ & \multirow[t]{2}{*}{$<0.001$} \\
\hline Type 2 & $37(10.4)$ & $208(9.5)$ & \\
\hline \multicolumn{4}{|l|}{ Etiology, $N(\%)$} \\
\hline HBV alone & $43(12.0)$ & $396(18.0)$ & \multirow{6}{*}{$<0.001$} \\
\hline $\mathrm{HCV}$ alone & $167(46.8)$ & $1,119(50.9)$ & \\
\hline Alcohol alone & $33(9.2)$ & $130(5.9)$ & \\
\hline Virus/alcohol & $48(13.4)$ & $146(6.6)$ & \\
\hline NAFLD/NASH & $24(6.7)$ & $138(6.3)$ & \\
\hline Miscellaneous ${ }^{\mathrm{b}}$ & $42(11.8)$ & $271(12.3)$ & \\
\hline
\end{tabular}

a Some data are missing.

b Autoimmune hepatitis, primary biliary cirrhosis, cryptogenic liver disease.
Table 3

Etiology of chronic liver disease in the 2001 and 2014 studies.

\begin{tabular}{lcc}
\hline Year of observation & 2001 study [3] & 2014 study \\
\hline Number of patients & 9,997 & 2,557 \\
With HBV alone, $N(\%)$ & $1,000(10.0)$ & $439(17.2)$ \\
With HBV plus alcohol, $N(\%)$ & $140(1.4)$ & $32(1.3)$ \\
With HBV plus HCV, $N(\%)$ & $141(1.4)$ & $32(1.3)$ \\
With HBV + alcohol + HCV, $N(\%)$ & $63(0.6)$ & $10(0.4)$ \\
With HCV alone, $N(\%)$ & $5,632(56.3)$ & $1,286(50.3)$ \\
With HCV plus alcohol, $N(\%)$ & $1,163(11.6)$ & $152(5.9)$ \\
With autoimmune hepatitis, $N(\%)$ & $76(0.8)$ & $60(2.3)$ \\
With primary biliary cholangitis, $N(\%)$ & $52(0.5)$ & $28(1.1)$ \\
With alcoholic liver disease, $N(\%)$ & $935(9.4)$ & $163(6.4)$ \\
With NAFLD/NASH, $N(\%)$ & $444(4.4)$ & $162(6.3)$ \\
With hemochromatosis, $N(\%)$ & $69(0.7)$ & $10(0.4)$ \\
With Wilson's disease, $N(\%)$ & $7(0.1)$ & $1(0.04)$ \\
With cryptogenic etiology, $N(\%)$ & $275(2.8)$ & $160(6.3)$ \\
With transitory hypertransaminasemia, & 0 & $22(0.9)$ \\
$\quad$ possibly iatrogenic, $N(\%)$ & & \\
\hline
\end{tabular}

HBV was responsible for chronic liver disease in 513 (20.2\%) cases, alone in 439 (17.2\%), associated with alcohol abuse in 32 (1.3\%), with HCV in $32(1.3 \%)$ and with HCV plus alcohol abuse in 10 (0.4\%). Of these $513(20.2 \%)$ patients, 61 (11.9\%) were anti-HDVpositive and 37 (7.2\%) were HBeAg-positive. HBV DNA was detected in $189(36.8 \%)$ of the $513 \mathrm{HBsAg}$-positive cases, with serum values lower than $2,000 \mathrm{IU} / \mathrm{mL}$ in 112 (59.3\%), between 2,000 and $20,000 \mathrm{IU} / \mathrm{mL}$ in $45(23.8 \%)$ and higher than $20,000 \mathrm{IU} / \mathrm{mL}$ in 32 (16.9\%) cases. Anti-HBc was detected in 724 (54.0\%) of 1,341 HBsAgnegative patients tested and anti-HBs in 368 (27.1\%) of 1,359 HBsAg-negative patients tested.

HCV was the etiologic agent of chronic liver disease in 50.3\% $(n=1,286 / 2,557)$ of the cases and in association with alcohol abuse in 5.9\% $(n=152)$. In all, HCV etiology accounted for $57.9 \%(n=1,480)$ of the cases [HCV alone in $50.3 \%(n=1,286), \mathrm{HCV}+$ alcohol in $5.9 \%$ $(n=152), \mathrm{HCV}+\mathrm{HBV}$ in $1.3 \%(n=32)$ and $\mathrm{HCV}+\mathrm{HBV}+$ alcohol in $0.4 \%$ $(n=10)$ ] (Table 3$)$. HCV RNA was detected in 947 (64.0\%) of the 1,480 anti-HCV-positive patients tested and HCV genotype was determined in 899 HCV-RNA-positive cases, of whom 140 (15.6\%) had HCV-genotype 1a, 409 (45.5\%) genotype 1b, 138 (15.4\%) genotype $2,78(8.2 \%)$ genotype $3,136(15.1 \%)$ genotype 4 and $2(0.2 \%)$ genotype 5 . Chronic liver disease was attributed to alcohol abuse in $6.4 \%(n=163)$ of the cases, to NAFLD/NASH in $6.3 \%(n=162)$, to autoimmune hepatitis in $2.3 \%(n=60)$, to primary biliary cholangitis in $1.1 \%(n=28)$, to hemochromatosis in $0.4 \%(n=10)$ and to Wilson's disease in $0.04 \%(n=1)$ (Table 2$)$. In addition, $6.3 \%$ of the cases $(n=160)$ were considered cryptogenic and $0.9 \%$ with transitory hyper-transaminasemia possibly iatrogenic $(n=22)$ (Table 3$)$.

Table 4 shows the clinical stage of the liver disease observed in the present investigation and in the 2001 study. Of the 492 subjects with HBV etiology and a definite diagnosis in the present study, $12.0 \%(n=59)$ were HBsAg inactive carriers, 59.3\% $(n=292)$ had chronic hepatitis, $24 \%(n=118)$ liver cirrhosis (compensated in $19.3 \%(n=95)$ and decompensated in $4.7 \%(n=23))$ and $4.7 \%(n=23)$ HCC, mostly cirrhotic. Of the 1,327 subjects with HCV etiology and a definite diagnosis observed in the present study, $4.4 \%(n=59)$ were subjects with persistently normal aminotransferases (PNALT), 57.2\% $(n=759)$ had chronic hepatitis, 31.9\% $(n=429)$ liver cirrhosis, (compensated in 22.4\% $(n=297)$ and decompensated in $9.5 \%$ $(n=126)$ ) and $6.5 \%(n=86)$ HCC, mostly cirrhotic.

Table 5 shows some demographic and clinical characteristics of four groups of patients we established based on the etiology and the availability of demographic and clinical information. HBV was the only etiologic agent in a group of 439 patients we named "the HBV group" and HCV the only etiologic agent in a group of 1,286 patients we named "the HCV group". The "Alcohol group" comprised 163 patients with long-lasting alcohol abuse as the only etiologic factor, 
Table 4

Stage of liver disease in the 2001 and 2014 studies.

\begin{tabular}{|c|c|c|}
\hline Year of observation & $2001[3]$ & 2014 \\
\hline All cases, total number (AC-TN) & 9,997 & 2,557 \\
\hline HBV, total number (HBV-TN) & 1,336 & 492 \\
\hline $\mathrm{HCV}$, total number (HCV-TN) & 6,498 & 1,327 \\
\hline Other etiology, total number (OE-TN) & 1,407 & 472 \\
\hline \multicolumn{3}{|l|}{ Diagnosis, $N(\%)$} \\
\hline HBV inactive carriers, $N$ (\% of HBV-TN) & $267(20.0)$ & $59(12.0)$ \\
\hline HCV subjects with PNALT, $N$ (\% of HCV-TN) & $474(7.3)$ & $59(4.4)$ \\
\hline Chronic hepatitis, $N$ (\% of AC-TN) & $6,250(62.5)$ & $1,342(52.5)$ \\
\hline $\mathrm{HBV}, N(\%$ of $\mathrm{HBV}-\mathrm{TN})$ & $768(57.5)$ & $292(59.3)$ \\
\hline $\mathrm{HCV}, N(\%$ of HCV-TN) & $4,555(70.1)$ & $759(57.2)$ \\
\hline Other etiologies, $N(\%$ of OE-TN) & $887(41.1)$ & $291(61.5)$ \\
\hline Liver cirrhosis, $N$ (\% of AC-TN) & $1,940(19.4)$ & $683(26.7)$ \\
\hline HBV, $N(\%$ of HBV-TN) & $248(18.6)$ & $118(24.0)$ \\
\hline $\mathrm{HCV}, N(\%$ of $\mathrm{HCV}-\mathrm{TN})$ & $1,225(17.3)$ & $423(31.9)$ \\
\hline Other etiologies, $N(\%$ of OE-TN) & $467(21.6)$ & $142(30.1)$ \\
\hline "compensated" cirrhosis, $N$ (\% of AC-TN) & $\mathrm{NA}$ & $503(19.7)$ \\
\hline $\mathrm{HBV}, N(\%$ of HBV-TN) & & $95(19.3)$ \\
\hline $\mathrm{HCV}, N(\%$ of $\mathrm{HCV}-\mathrm{TN})$ & & $297(22.4)$ \\
\hline Other etiologies, $N(\%$ of OE-TN) & & $111(23.5)$ \\
\hline "decompensated" cirrhosis, $N$ (\% of AC-TN) & NA & $180(7.0)$ \\
\hline $\mathrm{HBV}, N(\%$ of $\mathrm{HBV}-\mathrm{TN})$ & & $23(4.7)$ \\
\hline $\mathrm{HCV}, N(\%$ of $\mathrm{HCV}-\mathrm{TN})$ & & $126(9.5)$ \\
\hline Other etiologies, $N$ (\% of OE-TN) & & $31(6.6)$ \\
\hline HCC, $N(\%$ of AC-TN $)$ & $341(3.4)$ & $149(5.8)$ \\
\hline $\mathrm{HBV}, N(\%$ of HBV-TN) & $53(4.0)$ & $23(4.7)$ \\
\hline $\mathrm{HCV}, N(\%$ of $\mathrm{HCV}-\mathrm{TN})$ & $235(5.4)$ & $86(6.5)$ \\
\hline Other etiologies, $N$ (\% of OE-TN) & $53(2.4)$ & $40(8.5)$ \\
\hline Undefined diagnosis, $N$ (\% of AC-TN) & $756(7.6)$ & $265(10.4)$ \\
\hline
\end{tabular}

NA, not available.

whereas the "Virus/Alcohol group" comprised 194 patients with a multiple etiology, namely HBV plus alcohol or HCV plus alcohol. The mean age of the patients in these 4 etiologic groups was similar, while male sex predominated in the HBV group ( $n=288 / 65.6 \%$ ) and to a lesser extent in the HCV group ( $n=764 / 59.4 \%$ ). Patients in the Alcohol group and those in the Virus/Alcohol group more frequently than those in the HBV or HCV groups stated a primary school level of education; $p=0.006$. The diagnosis was based on liver biopsy in the majority of patients, more frequently for those in the HBV $(n=380 / 86.6 \%)$ and $\mathrm{HCV}(n=1,074 / 83.5 \%)$ groups than in the Alcohol $(n=119 / 73.0 \%)$ and Virus/Alcohol $(n=150 / 77.3 \%)$ groups $(p<0.001)$. Transient elastometry was performed in around one-third of the patients in each etiologic group. A higher rate of patients with liver cirrhosis and/or HCC was observed in the Alcohol group ( $n=58 / 39.4 \%$ ) and Virus/Alcohol group $(n=75 / 41.2 \%)$ than in the HBV and HCV groups ( $n=129 / 30.9 \%$ and $n=411 / 34.9 \%$, respectively; $p<0.001$ ). The highest rate of patients with decompensated cirrhosis was observed in the Alcohol group ( $n=24 / 16.3 \%$ ), followed by the Virus/Alcohol group ( $n=21 / 11.7 \%$ ), whereas much lower rates were observed in the HBV and HCV groups $(n=22 / 5.4 \%$ and $n=88 / 7.5 \%$, respectively; $p<0.001$ ).

\section{Discussion}

The above-mentioned prevalence surveys covering the period 1976-1989 were retrospective and did not consider HCV and multiple etiologies. Instead, the nationwide prevalence survey performed in 2001 and the present one performed in 2014 showed several structural similarities. Both studies were prospective, considered all etiologies and included tertiary and peripheral liver units throughout Italy. Consequently, the data from the present study provides an updated picture of chronic hepatitis in Italy, as well as an approximate evaluation of the etiologic variations over the last four decades and an accurate evaluation of the epidemiological and clinical changes from 2001 to 2014.

There was an impressive decrease in the rate of HBV-related cases from 1976 to 2001, as documented by the data from the reports covering the 1976-1981 (60.7\%), 1980-1989 (34.2\%) and 2001 (13.4\%) periods. Several factors can be considered responsible for this decline: the improvement in socio-economic conditions starting in the 60s and still ongoing in 2001, the intensive national mass media campaigns to limit the spread of AIDS in the 80s and 90s and the universal HBV vaccination that started in 1991 and had covered by 2001 the age classes $0-10$ and 12-21 [22-24]. In apparent contrast with the progressive extension of universal HBV vaccination, which by 2014 had covered all Italian subjects aged $0-34$, the rate of HBV-related cases had increased by 2014 to $20.2 \%$. Immigration from other countries does not account for this increase as demonstrated by the small rate $(5.7 \%)$ of immigrants enrolled in the present study and by their rate of HBsAg positivity (26.2\%). The increase in the rate of HBV-related cases observed in 2014 may be due to a referral bias, since after 2001 highly effective treatments (adefovir plus lamivudine, entecavir and tenofovir) for long-term suppression of HBV replication were introduced, possibly inducing the HBsAg-positive patients to go to the hospital centers issuing these drugs free of charge. Due to the prevalent epidemiological nature of the study, data on the treatment of CLD were not collected and this hypothesis, although likely, cannot be demonstrated. The hypothesis, however, is supported in part by the observation that liver cirrhosis and/or HCC had been diagnosed less frequently in the HBsAg-positive patients observed in 2001 than in those analyzed in 2014 ( $22.0 \%$ of 1,336 vs. $28.7 \%$ of 513 patients, $p=0.007)$.

The rate of HBeAg positivity in HBsAg-positive CLD has progressively declined in Italy from $58.4 \%$ detected in 356 HBsAg-positive patients with chronic persistent hepatitis studied from 1975 to

Table 5

Comparison of 2,082 patients according to etiology.

\begin{tabular}{|c|c|c|c|c|c|}
\hline & $\begin{array}{l}\text { HBV alone } \\
N=439\end{array}$ & $\begin{array}{l}\text { HCV alone } \\
N=1,286\end{array}$ & $\begin{array}{l}\text { Alcohol alone } \\
N=163\end{array}$ & $\begin{array}{l}\text { Virus/alcohol } \\
N=194\end{array}$ & $p$-value \\
\hline Age, years, media \pm SD & $58.1 \pm 13.5$ & $59.1 \pm 13.8$ & $60.6 \pm 12.0$ & $59.5 \pm 14.8$ & 0.2 \\
\hline \multicolumn{6}{|l|}{ Gender, $N(\%)$} \\
\hline Male & $288(65.6)$ & $764(59.4)$ & $88(54.0)$ & $106(54.6)$ & \multirow[t]{2}{*}{0.02} \\
\hline Female & $151(34.4)$ & $522(40.6)$ & $75(46.0)$ & $88(45.4)$ & \\
\hline \multicolumn{6}{|l|}{ Education, $N(\%)$} \\
\hline Primary school & $130(32.5)$ & $306(29.7)$ & $54(41.5)$ & $56(37.1)$ & \multirow[t]{4}{*}{0.006} \\
\hline Secondary school & $177(44.3)$ & $417(40.5)$ & $50(38.5)$ & $65(43.0)$ & \\
\hline High school/University & $93(23.3)$ & $306(29.7)$ & $26(20.0)$ & $30(19.9)$ & \\
\hline Missing, $N$ & 39 & 257 & 33 & 43 & \\
\hline \multicolumn{6}{|l|}{ Diagnosis, $N(\%)$} \\
\hline Absent/mild liver injury & $12(3.0)$ & $66(5.6)$ & $5(3.4)$ & $15(8.3)$ & \multirow[t]{6}{*}{$<0.001$} \\
\hline Chronic hepatitis & $267(66.1)$ & $703(59.6)$ & $84(57.1)$ & $91(50.6)$ & \\
\hline Compensated cirrhosis & $84(20.8)$ & $248(21.0)$ & $25(17.0)$ & $41(22.8)$ & \\
\hline Decompensated cirrhosis & $22(5.4)$ & $88(7.5)$ & $24(16.3)$ & $21(11.7)$ & \\
\hline $\mathrm{HCC}$ & $19(4.7)$ & $75(6.4)$ & $9(6.1)$ & $12(6.7)$ & \\
\hline Missing, $N$ & 35 & 106 & 16 & 14 & \\
\hline
\end{tabular}


1985 [25] to $20.1 \%$ found in a multicenter study performed in Italy in 1990 [26], to $13.6 \%$ observed in 2001 [4], to $11 \%$ in 2006-2007 [27] and to $7 \%$ in the present investigation in 2014. Universal HBV vaccination may be responsible, at least in part, for the decline observed after its first application in 1991, since all Italian subjects aged 34 or less had been vaccinated by 2014 and all HBV patients included in the present study aged 35 or more may have had enough time to seroconvert to HBeAg-negative. The mean age of the HBV patients observed in 2014 was 10 years older than that found in 2001, and, accordingly, with significantly higher rates of cirrhosis and HCC and significantly lower rates of inactive HBV carriage and chronic hepatitis.

The older age of HBV patients in the present study may also be a reason for the low rate of $\mathrm{HBsAg}$-positive patients circulating $\mathrm{HBV}$ DNA. Only one-third of the HBV patients were HBV-DNA positive, of whom, based on the current international guidelines [28,29], 59.3\% can be considered inactive HBV carriers (HBV DNA $<2,000 \mathrm{IU} / \mathrm{mL}$ ), $23.8 \%$ to have a progressive liver disease (HBV DNA between 2,000 and $20,000 \mathrm{IU} / \mathrm{mL}$ ), and $16.9 \%$ (HBV DNA $>20,000 \mathrm{IU} / \mathrm{mL}$ ) to be in an immune-tolerant phase. However, an inhibitory effect on HBV replication of drugs of high potency and high genetic barrier cannot be ruled out. Anti-HBc was detected in nearly half of the HBsAgnegative patients investigated and anti-HBs in nearly $30 \%$, clues to the wide spread of HBV infection in Italy in previous decades.

Multicenter prevalence surveys performed in Italy in 1987 [28], 1992 [29] and 1997 [30] showed a progressive decline in the proportion of HBsAg-positive patients with HDV infection $(23.4 \%$, $14.5 \%$ and $8.3 \%$, respectively), not or only slightly influenced by universal HBV vaccination started in Italy in the summer of 1991. HDV positivity was more or less stable from 2001 (9.7\%) to 2014 (11.9\%).

HCV infection was undoubtedly responsible for a majority of chronic liver diseases in Italy at least from 2001 [4], but a decreased impact of HCV etiology was registered from 2001 (69.9\%) to 2014 (57.9\%). We predicted this decline in our previous paper [4], based on the different rates of HCV etiology in incident and prevalent cases observed in 2001. This decline was also predicted by some studies in the general population showing an increasing rate of anti$\mathrm{HCV}$ positivity paralleling an increase in age [31-33]. In compliance with this, subjects with HCV infection in the present study were older (59.1 \pm 13.5 vs. $54.4 \pm 15.6)$ and presented increased rates of cases with a more severe disease than those in the 2001 survey. In 2014, efficient DAA-based HCV treatments were introduced in Italy, but their use was strongly limited by delays in the administrative practices for reimbursement. In addition, a referral bias similar to what we stated above for HBsAg-positive patients did not occur. Indeed, out of nearly 800,000 anti-HCV-positive subjects estimated in Italy, less than 30,000 had received a DAA-based treatment by the end of 2015 [8].

The impact of alcohol seems to have decreased from 2001 to 2014 , since its etiologic role, alone or in combination with other etiologies, was implicated in $23.0 \%$ of chronic liver diseases in 2001 and in $12.3 \%$ in 2014. A retrospective analysis of the reasons for this impressive decline is quite impossible. The percentage of patients with alcohol-related chronic liver diseases might have been underestimated both in the 2001 and 2014 surveys because of the negative stigma linked with alcohol abuse and of the patients' desires to keep on drinking, which may induce these patients to seek medical care only when their clinical condition has deteriorated. In addition, the possibility that not enough is being done in Italy to favor the access of patients with an alcohol-related liver disease to the structures of the National Healthcare System cannot be ruled out.

Information on incident or prevalent cases is not available. In this study, $84 \%$ of the population enrolled were outpatients, who are more likely to be incident cases than inpatients. The mean age of outpatients in the 2001 survey was 52.2 years, while the corresponding figure in the 2014 survey was 58.4 years $(p \leq 0.01)$. The mean age of inpatients in 2001 was 55.0 years, while that of inpatients in 2014 was 63.9 years $(p \leq 0.01)$. Thus a shift towards an older age affected both group of subjects (presumably also prevalent and incident cases), reflecting a shift towards an older age of the entire population.

Autoimmune hepatitis and primary biliary cholangitis were infrequently diagnosed both in the previous and present study, indicating that these diseases have only a marginal role in the setting of chronic liver diseases in Italy. However, a referral bias cannot be ruled out, since the availability of treatments against HBV and $\mathrm{HCV}$ infection is a strong incentive for patients with a viral etiology to refer to liver units assigning these expensive treatments free of charge. It is well known that NALFD is emerging as one of the most important causes of CLD in industrialized countries, where the prevalence of metabolic disorders is rapidly increasing due to unhealthy and sedentary lifestyles. A study performed in the general population in a geographic area in southern Italy showed that in as many as $24 \%$ of individuals with altered serum biochemical liver tests the probable cause was NAFLD [34]. In the hospital setting, the proportion of subjects with CLD due to NAFLD/NASH had increased by almost $50 \%$ over the 13 -year period, $4.4 \%$ in the 2001 study and 6.3\% in the present study (2014).

$\mathrm{HCV}$ remains the major etiologic factor of chronic hepatitis in Italy, a role, however, that will see a steady decline because of the spontaneous reduction in HCV endemicity in recent years and of the increasing use of the interferon-free DAA regimens to eradicate $\mathrm{HCV}$ infection.

Continuing universal HBV vaccination to all newborn babies will progressively increase the number of subjects protected against HBV. The impressive decrease in the rate of HBV-related cases from $60.7 \%$ in $1976-1981$ to $13.4 \%$ in 2001 is bound to continue, since the increase to $20.2 \%$ observed in 2014 most probably reflects the recent availability of new drugs for long-term suppression of HBV replication that are attracting numerous HBV patients to the liver units. Obviously, this situation is temporary and, by eliminating the infectivity of HBsAg-positive subjects, the administration of these drugs will further contribute to reducing the spread of HBV infection.

The low percentages of patients with autoimmune hepatitis, primary biliary cholangitis, Wilson's disease and iron overload are consolidated data reported in several studies over time. Instead, NALFD and NASH have been more frequently diagnosed in recent years, even though their prevalence may still be underestimated because those affected are often asymptomatic and tend not to seek medical care, thus generating a referral bias. These metabolic liver diseases warrant much more attention in the near future.

\section{Conflict of interest}

None declared.

\section{Funding}

An unrestricted grant for the study was provided by Gilead.

\section{References}

[1] Giusti G, Ruggiero G, Galanti B, et al. Chronic active hepatitis in Italy: a multicentric study on clinic and laboratory data of 1,154 cases. Hepato-Gastroenterology 1983;30:126-30

[2] Giusti G, Sagnelli E, Gallo C, et al. The etiology of chronic hepatitis in Italy: a multicentric study. Hepato-Gastroenterology 1994;41:397-400.

[3] Sagnelli E, Stroffolini T, Mele A, et al. The importance of HCV on the burden of chronic liver disease in Italy: a multicenter prevalence study on 9,997 cases. Journal of Medical Virology 2005;75:522-7.

[4] Stroffolini T, Sagnelli E, Mele A, et al. The etiology of chronic hepatitis in Italy: results from a multicenter national study. Digestive and Liver Disease 2004;36:829-33. 
[5] Sagnelli E, Stroffolini T, Mele A, et al. Chronic hepatitis B in Italy: new features of an old disease - approaching the universal prevalence of hepatitis B e antigennegative cases and the eradication of hepatitis D infection. Clinical Infectious Diseases 2008;46:110-3.

[6] Sagnelli E, Stroffolini T, Mele A, et al. Impact of comorbidities on the severity of chronic hepatitis B at presentation. World Journal of Gastroenterology 2012;18:1616-21.

[7] Stroffolini T, Sagnelli E, Mariano A, et al. Characteristics of HCV positive subjects referring to hospitals in Italy: a multicentre prevalence study on 6,999 cases. Journal of Viral Hepatitis 2006;13:351-4.

[8] http://www.easl.eu/research/our-contributions/clinical-practice-guidelines/ detail/recommendations-on-treatment-of-hepatitis-c-2015.

[9] Taal BG, Schalm SW, ten Kate FW, et al. Clinical diagnosis of primary biliary cirrhosis: a classification based on major and minor criteria. HepatoGastroenterology 1983;30:178-82.

[10] Alvarez F, Berg PA, Bianchi FB, et al. International autoimmune hepatitis group report: review of criteria for diagnosis of autoimmune hepatitis. Journal of Hepatology 1999;31:929-38.

[11] Adams PC, Chakrabarti S. Genotypic/phenotypic correlations in genetic hemochromatosis: evolution of diagnostic criteria. Gastroenterology 1998:114:319-23.

[12] Ferenci P, Caca K, Loudianos G, et al. Diagnosis and phenotypic classification of Wilson disease. Liver International 2003;23:139-42.

[13] Angulo P, Lindor KD. Non-alcoholic fatty liver disease. Journal of Gastroenterology and Hepatology 2002;17:S186-90.

[14] Gaiani S, Gramantieri L, Venturoli N, et al. What is the criterion for differentiating chronic hepatitis from compensated cirrhosis? A prospective study comparing ultrasonography and percutaneous liver biopsy. Journal of Hepatology $1997 ; 27: 979-85$

[15] Bruix J, Sherman M, Llovet JM, et al. Clinical management of hepatocellular carcinoma. Conclusions of the Barcelona-2000 EASL conference. European Association for the Study of the Liver. Journal of Hepatology 2001;35:421-30.

[16] Ishak K, Baptista A, Bianchi L, et al. Histological grading and staging of chronic hepatitis. Journal of Hepatology 1995;22:696-9.

[17] Bedossa P, Poynard T. An algorithm for the grading of activity in chronic hepatitis C. The METAVIR Cooperative Study Group. Hepatology 1996;24:289-93.

[18] Gamal S, Khaled Z. Ishak versus METAVIR: Terminology, Convertibility and Correlation with Laboratory Changes in Chronic Hepatitis C, Liver Biopsy. In: Takahashi H, editor. InTech. 2011. Available from: http://www.intechopen. com/books/liver-biopsy/ishak-versus-metavir-terminology-convertibilityand-correlation-with-laboratory-changes-in-chronic-h.

[19] Chon YE, Choi EH, Song KJ, et al. Performance of transient elastography for the staging of liver fibrosis in patients with chronic hepatitis B: a meta-analysis. PLOS ONE 2012;7:e44930.
[20] Wong VW, Vergniol J, Wong GL, et al. Liver stiffness measurement using XL probe in patients with nonalcoholic fatty liver disease. American Journal of Gastroenterology 2012;107:1862-71.

[21] Coppola N, Sagnelli C, Pisaturo M, et al. Clinical and virological characteristics associated with severe acute hepatitis B. Clinical Microbiology and Infection 2014;20:0991-7.

[22] Sagnelli E, Sagnelli C, Pisaturo M, et al. Epidemiology of acute and chronic hepatitis B and delta over the last 5 decades in Italy. World Journal of Gastroenterology 2014;20:7635-43.

[23] Mele A, Tosti ME, Mariano A, et al. Acute hepatitis B 14 years after the implementation of universal vaccination in Italy: areas of improvement and emerging challenges. Clinical Infectious Diseases 2008;46:868-75.

[24] Mele A, Mariano A, Tosti ME, et al. Acute hepatitis delta virus infection in Italy: incidence and risk factors after the introduction of the universal anti-hepatitis B vaccination campaign. Clinical Infectious Diseases 2007;44: e17-24.

[25] Giusti G, Galanti B, Gaeta GB, et al. Clinical presentation and natural history of chronic persistent hepatitis. A multicentre retrospective study on 1197 cases. Italian Journal of Gastroenterology 1991;23:111-8.

[26] Rapicetta M, di Nardo V, Rozera C, et al. HBV-DNA, HBeAg/anti-HBe serological status in hepatitis B chronic individuals from central Italy. Epidemiology and Infection 1990;104:511-7.

[27] Stroffolini T, Amasio PL, Sagnelli E, et al. Evolving clinical landscape of chronic hepatitis B: a multicenter Italian study. Journal of Medical Virology 2009;81:1999-2006.

[28] Sagnelli E, Stroffolini T, Ascione A, et al. The epidemiology of hepatitis delta infection in Italy, Promoting Group. Journal of Hepatology 1992;15:211-5

[29] Sagnelli E, Stroffolini T, Ascione A, et al. Decrease in HDV endemicity in Italy. Journal of Hepatology 1997;26:20-4.

[30] Gaeta GB, Stroffolini T, Chiaramonte M, et al. Chronic hepatitis D: a vanishing disease? An Italian multicenter study. Hepatology 2000;32(Pt 1):824-7.

[31] Guadagnino V, Stroffolini T, Rapicetta M, et al. Prevalence, risk factors, and genotype distribution of hepatitis $\mathrm{C}$ virus infection in the general population: a community-based survey in southern Italy. Hepatology 1997;26: 1006-11.

[32] Maio G, d'Argenio P, Stroffolini T, et al. Hepatitis C virus infection and alanine transaminase levels in the general population: a survey in a southern Italian town. Journal of Hepatology 2000;33:116-20.

[33] Di Stefano R Stroffolini T, Ferraro D et al. Endemic hepatitis C virus infection in a Sicilian town: further evidence for iatrogenic transmission. Journal of Medical Virology 2002;67:339-44.

[34] Pendino GM, Mariano A Surace P, et al. Prevalence and etiology of altered liver tests: a population-based survey in a Mediterranean town. Hepatology $2005 ; 41: 1151-9$ 